\title{
Simulation in Surgery
}

\author{
Tanwir Khaliq and Qurrat Al Ain Atif
}

Simulation based medical education (SBME) or simulation based training (SBT) has gradually paved its way into medical education. Its popularity and integration into curriculum has been initiated for over two decades ago. With emergency medicine, anesthesia, and obstetrics being the first fields to adapt this advancement, SBME is not shy even of psychiatry or general practice. 1

Medical education, particularly surgeons, have been taught through the Halstedian model of surgical training, that is learning the surgical skills through apprenticeship - "See one, do one, teach one". ${ }^{2}$ After decades of such practice, concerns regarding ethical issues of patients being treated by trainees' cost of training and long learning and teaching hours, lead to utilization of simulation in healthcare education. European working time regulation has significantly affected and shortened the surgical exposure of a trainee, which could only be dealt with by accommodating for it by simulation. ${ }^{3}$ Moreover, the trend toward minimally invasive surgery has prompted the incorporation of SBT to reduce learning curve for trainees. ${ }^{4}$ Surgical training has shifted from operating room to surgical skills suites. So, trainees are well adept in varying degrees of complex and complicated procedures as well as minimally invasive techniques in a shorter period of time without patients being at harm, in agreement with Hippocratic principle "First, do no harm".

Simulation is described as an educational technique in which "real-world" concepts are integrated into scenarios utilizing low to high fidelity tools to reenact the "realworld" situations. ${ }^{5}$ Initially designed and utilized in the aviation, spatial engineering, and military sectors, its importance in the healthcare sector was established. The aim was to minimize the number of patient deaths and disabilities resulting from medical errors. ${ }^{6}$

The use of simulation can be dated back to the $18^{\text {th }}$ Century where obstetric model mannequins were designed to teach; and later, in the middle ages, animals were used to train surgical skills.

Nowadays, SBME has become cornerstone in medical education for teaching technical and non-technical skills in a safe, non-threatening, and controllable environment.

Pakistan Institute of Medical Sciences, Islamabad, Pakistan

Correspondence: Prof Tanwir Khaliq, Department of Medical

Education, Federal Medical and Dental College Pakistan

Institute of Medical Sciences, Islamabad, Pakistan

E-mail: ktanwir@hotmail.com

Received: October 29, 2018; Accepted: November 10, 2018
SBME includes "devices, trained persons, lifelike virtual environments, and contrived social situations that mimic problems, events or conditions that arise in professional encounters". Current examples include the CPR simulator, Harvey cardiology patient simulator, Resusci Anne. ${ }^{7}$

Simulation may occur in many locations including: dedicated wet or dry labs in specialist simulation laboratories, within working theatres, and even in the trainee's home either using cheap, basic jigs, or increasingly sophisticated computer equipment.

Simulators in surgery are categorized as either organic (high fidelity) or inorganic (low fidelity). Organic simulators include live animals and human cadavers. Inorganic simulators comprise of virtual reality simulators, synthetic bench models, low fidelity laparoscopic trainers, trainers for new surgical techniques, i.e. single incision laparoscopic surgery (SILS), natural orifice transluminal endoscopic surgery (NOTES) and robotic surgery simulators. Apart from these are simulators for teaching non-technical surgical skills, namely, professionalism, communication skills, team work, and leadership/followership, interdisciplinary and interprofessional coordination. These criteria are set forth by Accreditation Council for Graduate Medical Education (ACGME) and Institute for International Medical Education as requisite components of medical competence.

Simulation has its future not only in training but also in selecting and assessing trainees. Based on dexterity and procedural skills, trainees can be selected to various surgical specialties. Although non-technical surgical skills are accessed via established simulation models; for example, OSCE, the integration of assessing surgical skills using simulators is controversial. The main reason behind this is the restriction of surgical training to simulation suites.

Keeping in pace with international standards and advancements, simulation centers have also been established in Pakistan. A number of postgraduate and under-graduate medical institutes are developing simulation laboratories in their centers. These centers are catering excellent opportunities for trainees in learning and improving their skills. Some institutes are offering training and assessment in basic life support, advanced cardiac life support, and advanced trauma life support with certification and accreditation; and others are using as assessment tools in OSCE and clinical examinations. 8,9

Unfortunately, the high cost and high resource utilization simulation training make its availability limited in Pakistan's 
teaching hospitals. Despite being proven to be an excellent teaching modality leading to better learning experience in various studies, it is still not integrated into undergraduate and postgraduate medical curriculum in Pakistan. ${ }^{10}$

Addressing ethical concerns of patient safety, cost effectiveness of training, and restricted working hours, all have led to a paradigm shift in surgical training. Simulation has substantiated to be an integral tool in medical education in terms of teaching technical as well as non-technical skills, selecting and assessing trainees. There is still huge potential for research in this field of healthcare training to justify its safety, transferability, retain ability, cost effectiveness and methods to reduce its cost, as well as patient reported outcomes. Nonetheless, simulation shows a promising future and thus should become a fundamental part of medical education.

\section{REFERENCES}

1. Walsh K. The future of simulation in medical education. J Biomed Res 2015; 29: 259-60.

2. Montbrun SL, MacRae H. Simulation in surgical education. Clin Colon Rectal Surg 2012; 25:156-65.

3. Khera G, Milburn J, Hornby S, Malone P. Simulation in surgical training. A statement from The Association of Surgeons in Training. November 2011.

4. Kneebone R, Aggarwal R. Surgical training using simulation. BMJ 2009; 338:b1001

5. Williams B, Abel C, Khasawneh E, Ross L, Levett-Jones T. Simulation experiences of paramedic students: a cross-cultural examination. Adv Med Educ Pract 2016; 7:181-6.

6. Jones F, Passos-Neto E, Braghiroli OFM. Simulation in medical education: Brief history and methodology. Princ Pract Clin Res 2015; 1:56-63.

7. Sørensen JL, Navne LE, Martin HM, Ottesen B, Albrecthsen CK, Pedersen BW et al. Clarifying the learning experiences of healthcare professionals with in situ and off-site simulationbased medical education: a qualitative study. BMJ Open 2015; 5:e008345.

8. Zafar A. Minimal access surgery in Pakistan: Role of virtual reality simulation in training. Isra Med J 2014; 6:183-4.

9. Shafiq Z, Mufti TS, Qayum I. Role of clinical skill center in undergraduate medical education: Initial experience at Rehman Medical College Peshawar. J Pak Med Assoc 2017; 67:73-6.

10. Shah N, Baig L, Shah N, Hussain R, Aly SM. Simulation-based medical education; Teaching normal delivery on intermediate fidelity simulators to medical students. J Pak Med Assoc 2017; 67:1476-81 